\title{
HIGHLY RESPONSIVE HEADING CONTROL OF UNDERACTUATED MODIFIED BLUEROV USING SLIDING-MODE CONTROLLER
}

\author{
M. F. YAHYA and M. R. ARSHAD \\ Underwater, Control and Robotics Group, \\ School of Electrical and Electronic Engineering, \\ Universiti Sains Malaysia, Engineering Campus, \\ Nibong Tebal, Pulau Pinang, 14300, Malaysia. \\ E-mail: mohdfaid85@gmail.com,eerizal@usm.my \\ www.usm.my
}

\begin{abstract}
The control of Autonomous Underwater Vehicle (AUV) presents some challenging tasks such as dealing with the nonlinearity of AUV dynamics, coupling effects, and taking into consideration the hydrodynamics uncertainties and disturbances such as current. One of the more robust control method for controlling a nonlinear system is Sliding-Mode Control (SMC). In literature, SMC had been used to control the depth and yaw of an underactuated AUV. However, the switching term of the SMC had to be tuned to get a proper balance between performance and robustness and it has low responsiveness to track desired reference signal. This paper introduces the usage of smoothing filter to make the SMC more responsive to track desired heading motion. The methodology starts with deriving heading model of an AUV, then estimating the parameters in the AUV model, followed by designing the heading controller based on SMC, and finally optimizing the controller parameters. The design model is based on underactuated modified BlueROV. Results shown that when the reference heading signal is smoothed, the SMC is able to achieve $95.55 \%$ responsiveness compared to only just $78.05 \%$ responsiveness without smoothing. The thrusts produced by the AUV is also less strained when the smoothing filter is applied. Therefore, to get a highly responsive SMC and to preserve the longevity of the thrusters of an AUV, a smoothing filter had to be considered in controller design of an underactuated AUV.
\end{abstract}

\section{Introduction}

According to National Oceanic and Atmospheric Administration (NOAA), over 1000 ship wrecks lie off at the Florida Keys. To investigate each one of them manually is a tedious job which is better performed by Autonomous Underwater Vehicle (AUV). Apart from the shipwreck investigation, an AUV has the potential to perform underwater cave exploration and studying coral reefs, all of which requires sophisticated motion control. The control of AUV presents some challenging tasks such as dealing with the nonlinearity of AUV dynamics, coupling effects, and taking into consideration the hydrodynamics uncertainties and disturbances such as flow of current.

One of the more suitable controller for controlling a nonlinear system is Sliding-Mode Control (SMC). The SMC aims to make a sliding variable goes to zero as time approaches infinity. The sliding variable is defined as the tracking error times eigenvector of a closed-loop system. SMC had been used on AUV for depth control [1], yaw control [2,3], tracking control under ocean currents [4], and tracking control for under-actuated system [5]. Apart from its normal usage, there are several improvements made for controlling an AUV. In one study, the switching term of SMC was tuned using extreme learning machine (ELM) [6]. In another study, a combination of method consisting of backstepping and SMC are used to control an autonomous underwater glider [7]. In addition, this combination of method has been used on an AUV with a neural network added on top of the controller to tune SMC switching term [8]. Basically, higher switching term gain adds robustness to the controller at the cost of performance while lower switching term gain make the controller performs better but lacks robustness to handle disturbances. 
In all, SMC has a simple design principle yet very robust to handle model uncertainties and unexpected disturbances. On the other hand, there are some issues such as configuring the tuning parameter of switching term gain to get the desirable balance between performance and robustness, and it has low responsiveness to desired reference signal.

In this paper, the objective is to design and develop a responsive and robust controller based on SMC for an underactuated hovering AUV. The focus of the controller is on heading motion. Heading refers to the capability of the robot to turn left or right and it is one of the most important motion to control. The scope covers the applicability of the control only for a modified BlueROV prototype and some of the test was conducted at diving pool with calm water instead of at sea.

\section{Methodology}

The process of designing a proper heading control system for an AUV starts with modeling. Modeling means a simple description of a system. In this research, the AUV's heading is described by its heading equation of motion. After the heading equations of motion had been derived, there would be some parameters in the model that is hard to determine due to the nonlinearity of hydrodynamics properties. So, estimation of these unknown parameters by system identification is required. Next, a heading control system based on SMC is designed. Then, the SMC controller parameters are optimized to get desired response. Once the controller had been optimized, the response of the robot is evaluated based on certain metrics. In all, the flowchart of the methodology is shown in Figure 1.

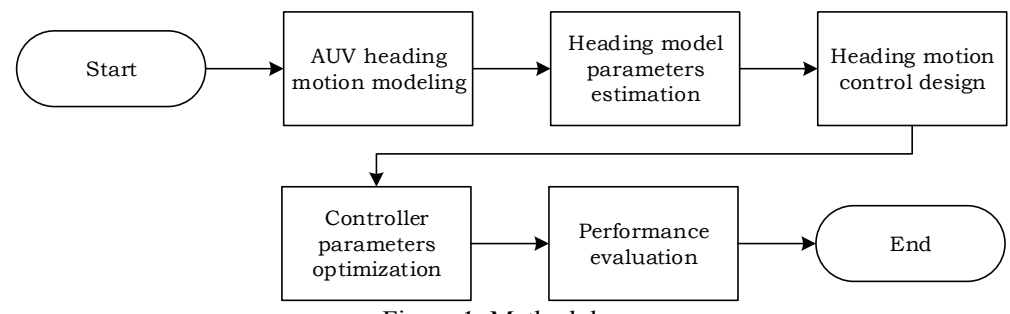

Figure 1. Methodology

\subsection{Heading Model of AUV}

The prototype to be modeled is a modified BlueROV. The modified BlueROV is chosen because it is lightweight, small-sized, and has high maneuverability. Figure 2 shows the design of the robot as well as motion variables, $u, v, w, p, q$, and $r$ in $\mathrm{m} / \mathrm{s}$. Although the main usage of the robot is as a Remotely-Operated Vehicle (ROV), it is used primarily as an AUV in this study. Focus is also given to thrusters $\mathrm{T}_{3}$ and $\mathrm{T}_{4}$ which produces heading motion.

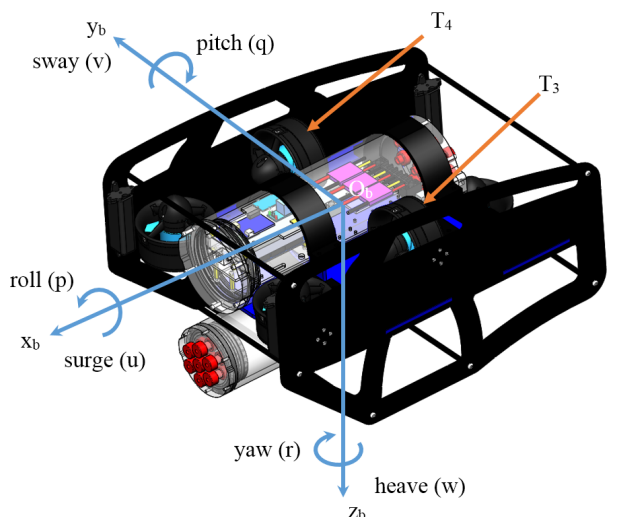

Figure 2. Modified BlueROV design with motion variables 
The modeling derivation of the robot is based on [9]. The heading model of the robot in state space is given as follow

$$
\left[\begin{array}{c}
\dot{v}(t) \\
\dot{r}(t) \\
\dot{\psi}(t)
\end{array}\right]=\left[\begin{array}{ccc}
\frac{Y_{v}}{m-Y_{\dot{v}}} & \frac{Y_{r}}{m-Y_{\dot{v}}} & 0 \\
\frac{N_{v}}{I_{z}-N_{\dot{r}}} & \frac{N_{r}}{I_{z}-N_{\dot{r}}} & 0 \\
0 & 1 & 0
\end{array}\right]\left[\begin{array}{c}
v(t) \\
r(t) \\
\psi(t)
\end{array}\right]+\left[\begin{array}{c}
0 \\
\frac{1}{I_{z}-N_{\dot{r}}} \\
0
\end{array}\right] N(t)
$$

where $v$ is swaying speed, $r$ is yawing speed, $\psi$ is yawing angle, $Y_{v}$ is linear damping force coefficient with respect to swaying, $m$ is mass of the modified BlueROV, $Y_{v}$ is added mass with respect to swaying, $Y_{r}$ is linear damping force coefficient with respect to yawing, $N_{v}$ is linear damping force coefficient with respect to swaying, $I_{z}$ is inertia about zb-axis, $N_{\dot{r}}$ is added mass with respect to yawing, $N_{r}$ is linear damping force coefficients with respect to yawing, and $N$ is moment about $\mathrm{Zb}$-axis produces by thrusters $\mathrm{T}_{3}$ and $\mathrm{T}_{4}$ (both of $\mathrm{T} 100$ model thruster).

\subsection{Parameter Estimation of Heading AUV Model}

Although the equations of motion had been derived, some of the components in the equations are unknown. The unknown components consist of hydrodynamics and inertial parameters. These unknown parameters need to be estimated using system identification. Gray-box system identification is used because the modeling and prototype are available. The process to estimate parameters based on gray-box system identification is firstly to perform experiment, secondly to run simulation, and thirdly to estimate parameters by comparing the 2 sets of collected data.

For experimentation, let the AUV submerged in a pool for 1 meter and let it stabilized (neutrally buoyant). Then, starts to record the yawing angle, $\psi$. When $t$ is equal to 2,8 , and 14 seconds, $\mathrm{T}_{3}$ and $\mathrm{T}_{4}$ are supplied with an instantaneous force of $1.37 \mathrm{~N}$ but with different direction so that the AUV produces rotation about $\mathrm{zb}$-axis. The duration of test is set to $20 \mathrm{~s}$ and the experiment is repeated 3 times to get average value. Figure 3 shows the illustration of the experiment.

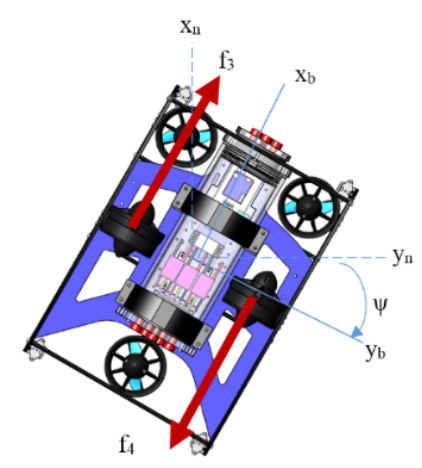

Figure 3. System identification experiment for heading

For simulation, the known value is the mass $m$ which is $6.2 \mathrm{~kg}$ and the initially guessed values are $Y_{r}=0.1 \mathrm{~kg} / \mathrm{s}, Y_{v}=0.1 \mathrm{~kg} / \mathrm{s}, Y_{\dot{v}}=0.1 \mathrm{~kg}, I_{z}=0.015 \mathrm{~kg} \mathrm{~m}^{2} / \mathrm{s}^{2}, N_{v}=0.1 \mathrm{~kg} \mathrm{~m} / \mathrm{s}, N_{r}=1.5$ $\mathrm{kg} \mathrm{m} / \mathrm{s}$, and $N_{\dot{r}}=0.5 \mathrm{~kg} \mathrm{~m} / \mathrm{s}^{2}$. The simulation is conducted for $20 \mathrm{~s}$ similar to experiment duration and the yawing angle, $\psi$ is recorded.

Based from the 2 yawing angle data sets (experiment and simulation), the initially guessed values are estimated using nlgreyest function from MATLAB and the estimation is further improved using pem function. The final estimated values are $Y_{r}=-1 \mathrm{~kg} / \mathrm{s}, Y_{v}=-10 \mathrm{~kg} / \mathrm{s}, Y_{\dot{v}}=6$ $\mathrm{kg}, I_{z}=0.015 \mathrm{~kg} \mathrm{~m}^{2} / \mathrm{s}^{2}, N_{v}=10 \mathrm{~kg} \mathrm{~m} / \mathrm{s}, N_{r}=1.3 \mathrm{~kg} \mathrm{~m} / \mathrm{s}$, and $N_{\dot{r}}=0.5 \mathrm{~kg} \mathrm{~m}^{2} / \mathrm{s}^{2}$. Note that the Normalized Root Mean Squared Error (NRMSE) percentage for simulation, nlgreyest function, and pem function are $9.3 \%, 88.6 \%$, and $92.2 \%$ respectively. 


\subsection{Heading Motion Control Design}

The block diagram of the designed heading control system using SMC is shown in Figure 4. There are four significant blocks denoted by the bolded text and number.

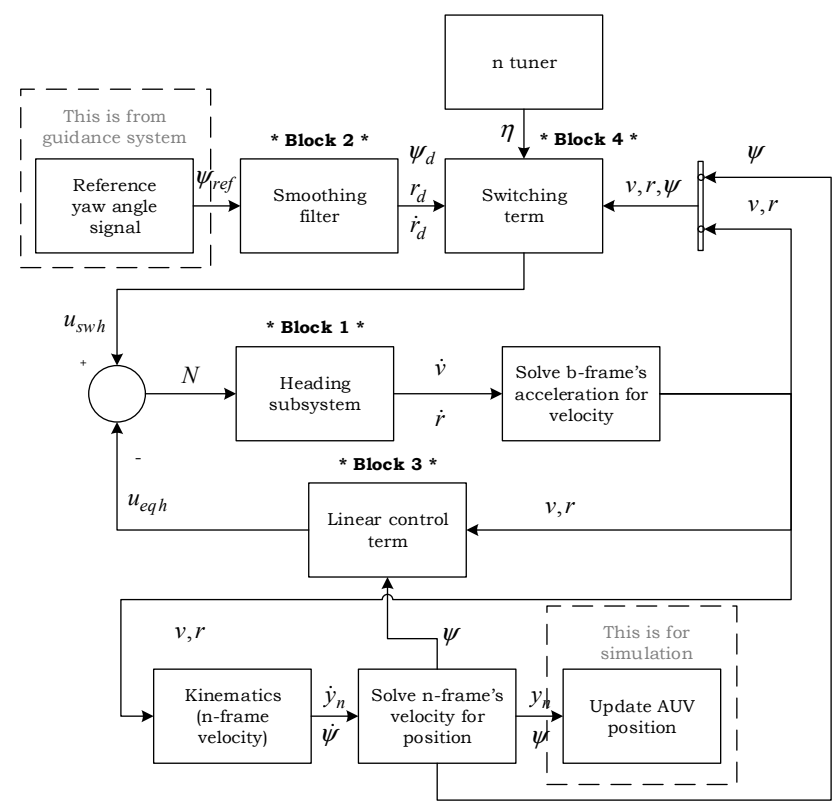

Figure 4. Block diagram of the designed heading control system

The first block (Block 1) is about heading subsystem and the equation is given as in Eq. (1). Then, the second block (Block 2) is about the smoothing filter. It smooths the reference input so that the signal appears analogous rather than digital. The smoothing filter is of second order mass-spring-damper system given by

$$
\ddot{\psi}_{d}+2 \zeta \omega_{o} \dot{\psi}_{d}+\omega_{o}^{2} \psi_{d}=\omega_{o}^{2} \psi_{r e f}
$$

with

$$
\begin{aligned}
& \dot{\psi}_{d}=r_{d} \\
& \ddot{\psi}_{d}=\dot{r}_{d}
\end{aligned}
$$

where $\psi_{\text {ref }}$ is reference yaw angle, $\psi_{d}$ is desired yaw angle, $r_{d}$ is desired yaw angular velocity, and $\dot{r}_{d}$ is desired yaw angular acceleration. The damping ratio $\zeta$ is set to 1 and the natural frequency $\omega_{o}$ is set to 2 to get a combination of critically damped and quicker response signal.

Next, Block 3 is a linear control term block. The input to the block is state variables consisting of sway velocity $v$, yaw angle rate $r$ and yaw angle $\psi$. So, there will be three parameters for linear control term such that

$$
\mathbf{k}_{h}=\left[k_{h 1}, k_{h 2}, k_{h 3}\right]^{T}
$$

where $k_{h l}, k_{h 2}$ and $k_{h 3}$ are poles placed on the closed-loop system to control the $v, r$ and $\psi$ terms respectively. The output for the equivalent linear controller block is 


$$
u_{e q h}=\mathbf{k}_{h}^{T} \mathbf{x}_{h}=\left[k_{h 1}, k_{h 2}, k_{h 3}\right]\left[\begin{array}{c}
v \\
r \\
\psi
\end{array}\right]
$$

The objective of the linear controller block is not just to control the state variables, but to also find the eigenvalues for the switching term. This is done by decomposing the eigenvalues of a closed-loop system matrix. The closed-loop system matrix for the heading subsystem $\mathbf{A}_{c h}$ with the respective linear controller can be modeled by

$$
\mathbf{A}_{c h}=\left[\begin{array}{ccc}
a_{11} & a_{12} & 0 \\
a_{21}-b_{h} k_{h 1} & a_{22}-b_{h} k_{h 2} & -b_{h} k_{h 3} \\
0 & 1 & 0
\end{array}\right]
$$

Let $\mathbf{h}_{h}$ be an eigenvector to the heading switching term and according to [9]

$$
\lambda_{h} \mathbf{x}_{h}^{T} \mathbf{h}_{h}=0 \text { if } \mathbf{h}_{h} \text { is a right eigenvector of } \mathbf{A}_{c h}^{T} \text { for } \lambda_{h}=0
$$

where $\lambda_{h}=0$ is an eigenvalue for the pure integrator of $r$ producing $\psi$. So, $\mathbf{h}_{h}$ is found by decomposing eigenvalues based from $\mathbf{A}_{c h}^{T}$. Therefore,

$$
\mathbf{h}_{h}=\left[h_{h 1}, h_{h 2}, h_{h 3}\right]^{T}
$$

are to be optimized SMC parameters.

The final block or Block 4 is about switching term. The output of the switching block is given as follow

$$
\begin{aligned}
u_{s w h} & =\left(\mathbf{h}_{h}^{T} \mathbf{b}_{h}\right)^{-1}\left[\mathbf{h}_{h}^{T} \dot{\mathbf{x}}_{d}-\mathbf{h}_{h}^{T} \hat{\mathbf{f}}(\mathbf{x}, t)-\eta \operatorname{sgn}\left(s_{h}\right)\right] \\
& =\frac{1}{h_{h 2} b_{h}}\left[h_{h 2} \dot{r}_{d}+h_{h 3} \dot{\psi}_{d}-\eta \tanh \left(s_{h}\right)\right], \eta>0
\end{aligned}
$$

where $s_{h}$ is the heading sliding surface such that

$$
s_{h}=\mathbf{h}_{h}^{T} \tilde{\mathbf{x}}=\mathbf{h}_{h}^{T}\left(\mathbf{x}_{h}-\mathbf{x}_{h d}\right)=h_{h 1} v+h_{h 2}\left(r-r_{d}\right)+h_{h 3}\left(\psi-\psi_{d}\right)
$$

Therefore, the heading thrust needed to track desired yaw angle $\psi_{d}$ is

$$
N=-u_{e q h}+u_{s w h}=-k_{h 1} v-k_{h 2} r+\frac{1}{h_{h 2} b_{h}}\left[h_{h 2} \dot{r}_{d}+h_{h 3} \dot{\psi}_{d}-\eta \tanh \left(s_{h}\right)\right]
$$

$N$ can be transformed into actuator forces by summing the resultant forces and moments so that

$$
\begin{aligned}
f_{3}+f_{4} & =0 \\
-f_{3} l_{y 3}+f_{4} l_{y 4} & =N
\end{aligned}
$$

where $l_{y 3}=l_{y 4}=0.11 \mathrm{~m}$ are distance from origin of body frame to $\mathrm{T}_{3}$ and $\mathrm{T}_{4}$ origins respectively.

\subsection{Controller Parameters Optimization}

The controller parameters are optimized using trial and error method by means of brute force. The parameters values tested are from -1 to -40 with decrement of -1 . Combination of parameters values which produces minimum sum of absolute error is selected. So, let the waypoint be 


$$
\psi_{\text {ref }}=\left\{\begin{array}{cc}
0 & t=0 \\
\pi / 4 & 0<t \leq 6 \\
\pi / 9 & 6<t \leq 12 \\
-\pi / 18 & 12<t \leq 18 \\
-\pi / 4 & 18<t \leq 24 \\
0 & 24<t \leq 30
\end{array}\right.
$$

Figure 5 shows the response of heading using the worst, mediocre and best (optimized) controller parameters values. For the optimized controller parameters values $\left(h_{l}=0, h_{2}=-39\right.$, $h_{3}=-40$ ), the sum of absolute error is 2.98 . Note that the switching gain $\eta$ is fixedly set to 100 .

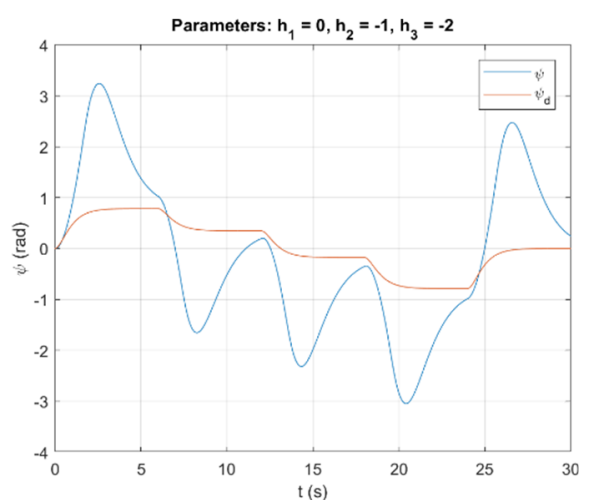

(a)

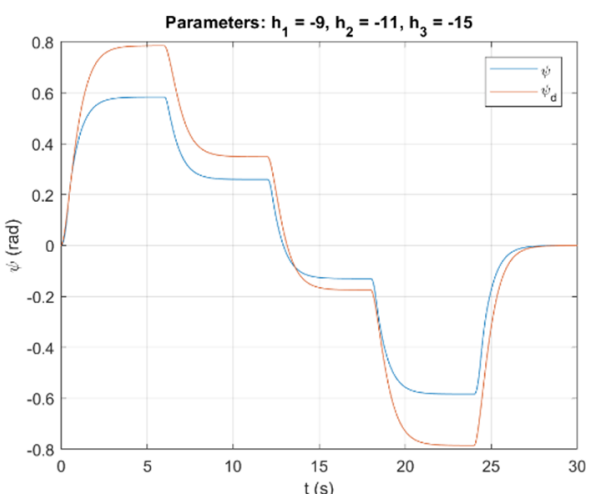

(b)

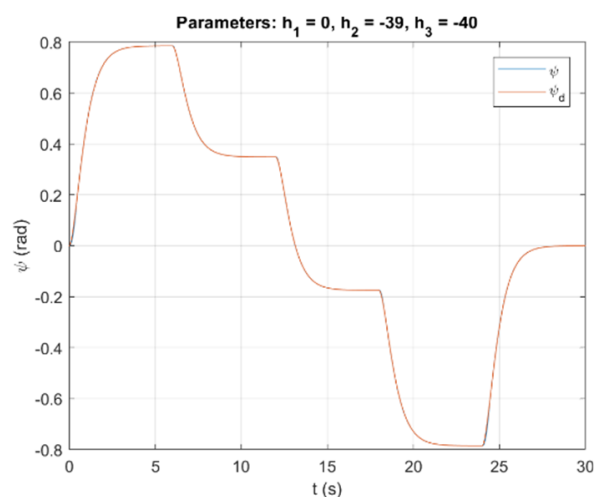

(c)

Figure 5. Heading responses using different sets of controller parameters

\section{Results and Discussions}

The performance metric used to conduct the analysis of the results is responsiveness to unit step input. The formula to calculate the responsiveness to unit step is given by

$$
R=100\left(1-\frac{1}{N} \sum_{n_{s}=1}^{N}\left|\frac{\psi_{d}\left(n_{s}\right)-\psi_{a}\left(n_{s}\right)}{\psi_{d}\left(n_{s}\right)}\right|\right)
$$

where $n_{s}$ is the sample number, $N$ is the total number of samples, and $\psi_{d}\left(n_{s}\right)$ and $\psi_{a}\left(n_{s}\right)$ are the desired and actual yaw values for sample $n_{s}$ respectively. The formula for unit step is given as

$$
\psi_{d}\left(n_{s}\right)=1
$$


The reference signal can be smoothed to create another type of desired signal. The desired smoothed signal using mass-spring-damper system as suggested during the design of control system is given as

$$
\psi_{d}\left(n_{s}-2\right)+2 \zeta \omega_{o} \psi_{d}\left(n_{s}-1\right)+\omega_{o}^{2} \psi_{d}\left(n_{s}\right)=\omega_{o}^{2} \times 1
$$

So, there are two input signals used as tracking sources for the designed controller. Figure 6 shows the responses of the robot when the heading controller tracks the desired reference and desired smoothed signals. Also shown are the thrusts needed to perform the maneuver. Note that the lower limit of thrust is set to $-5 \mathrm{~N}$ and upper limit of thrust is set to $5 \mathrm{~N}$.

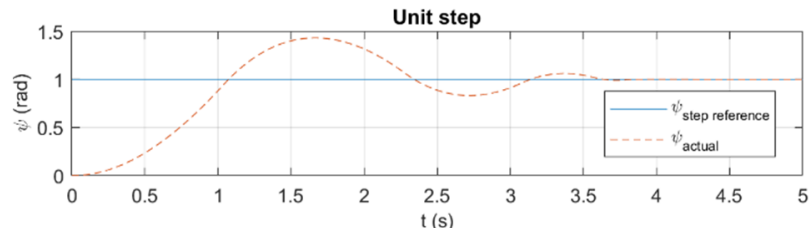

(a)

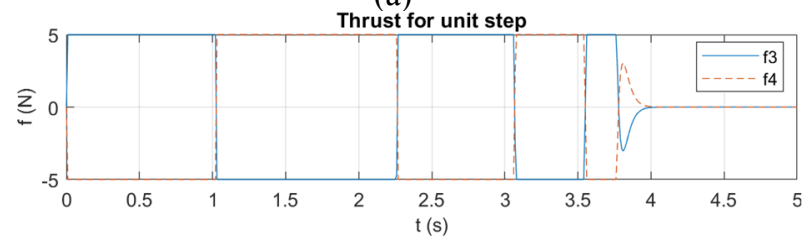

(b)

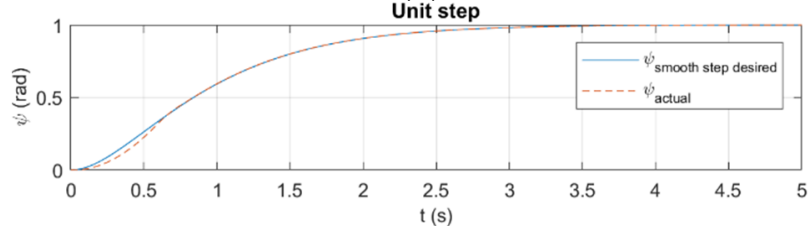

(c)

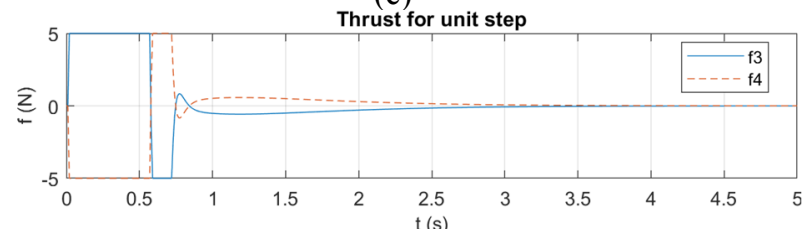

(d)

Figure 6. Response of robot and thrust produced by SMC for heading control, (a) and (b) for desired reference input signal, (c) and (d) for desired smoothed input signal

Figure 6 (a) shows the response of the robot based from the thrust produced by SMC for heading using desired reference input signal. Based from the figure, for unit step signal, the response is oscillating at the beginning, but became steady after $4 \mathrm{~s}$. It is observed that the thrusts as in Figure 6 (b) that produce this response are alternating steeply at about $1 \mathrm{~s}, 2.25 \mathrm{~s}, 3.1 \mathrm{~s}, 3.6 \mathrm{~s}$, and $3.75 \mathrm{~s}$ before they stabilize. Then, Figure 6 (c) shows the response of the robot based on desired smoothed input signal using SMC. There are no overshoot and the controller shows excellent tracking performance. The corresponding thrust as shown in Figure 6 (d) changes gradually instead of steeply for a longer period of time compared to Figure 6 (b). Evidently, from Figure 6 (a) and (c), the designed SMC produces stable heading output response patterns. In all, the responsiveness of the heading based on the desired reference input is $78.05 \%$ while desired smoothed input gives $95.55 \%$. In addition, using the desired reference signal strains the thruster more while using the desired smoothed signal preserves the thruster better. Therefore, 
it is necessary to smooth the reference input signal so that the AUV performs better responsively and to preserve the longevity of the thrusters.

\section{Conclusions and Future Works}

In conclusion, a heading control system based on SMC had been designed and developed for a modified BlueROV. In order to make the controller more responsive, a smoothing filter is introduced to the reference signal so that the controller is able to track the signal with reduced tracking error. Quantitatively, the controller shown to have 95.55\% responsiveness with smoothing filter and $78.05 \%$ responsiveness without the smoothing filter. Qualitatively, the controller has excellent responsiveness to follow smoothed reference signal and acceptable responsiveness to follow non-smoothed reference signal.

The future works could include updating the estimated parameters from the heading model with sea water instead of pool water or introducing lateral disturbance (current) or developing an algorithm to further optimize controller parameters to get an even better heading response.

\section{Acknowledgement}

This research is fully sponsored by the Kementerian Pendidikan Malaysia (KPM), under the Fundamental Research Grant Scheme (FRGS). Account No.: 1001/PELECT/6071346. In addition, the first author is grateful to both Universiti Teknikal Malaysia Melaka (UTeM) and KPM for giving allowances during his study.

\section{References}

1. Y. S. Siang and M. R. Arshad, "Sliding mode depth control of a hovering autonomous underwater vehicle," in IEEE International Conference on Control System, Computing and Engineering (ICCSCE), 2015, pp. 435-440.

2. Y. V. Medina, A. F. Tkachova, L. H. Santana, and P. J. P. Entenza, "Yaw Controller in Sliding Mode for Underwater Autonomous Vehicle," IEEE Latin America Transactions, vol. 14, pp. 1213-1220, 2016.

3. H. Akçakaya, H. A. Yildiz, G. Sağlam, and F. Gürleyen, "Sliding mode control of autonomous underwater vehicle," in International Conference on Electrical and Electronics Engineering - ELECO, 2009, pp. II-332-II-336.

4. M. Farhan, A. I. Bhatti, W. A. Kamal, and I. K. Yousafzai, "Sliding mode based MIMO control of autonomous underwater vehicle," in 11th Asian Control Conference (ASCC), 2017, pp. 2899-2904.

5. T. Elmokadem, M. Zribi, and K. Youcef-Toumi, "Trajectory tracking sliding mode control of underactuated AUVs," Nonlinear Dynamics, vol. 84, pp. 1079-1091, 2016/04/01 2016.

6. D. Wang, Y. Shen, Q. Sha, G. Li, J. Jiang, B. He, et al., "Controller design of an autonomous underwater vehicle using ELM-based sliding mode control," presented at the OCEANS Anchorage, AK, USA, 2017.

7. M. Mat-Noh, M. R. Arshad, R. Mohd-Mokhtar, and Q. Khan, "Back-stepping integral sliding mode control (BISMC) application in a nonlinear autonomous underwater glider," in IEEE 7th International Conference on Underwater System Technology: Theory and Applications (USYS), 2017, pp. 1-6.

8. Z. Chu and D. Zhu, "3D path-following control for autonomous underwater vehicle based on adaptive backstepping sliding mode," in IEEE International Conference on Information and Automation, 2015, pp. 1143-1147.

9. T. I. Fossen, Handbook of Marine Craft Hydrodynamics and Motion Control, 1 ed. United Kingdom: John Wiley \& Sons, 2011. 
